\title{
Commentary
}

\section{Action Plan for a Healthy Agriculture, Healthy Nutrition, Healthy People}

\author{
Artemis P. Simopoulos ${ }^{\mathrm{a}}$ Ole Faergeman ${ }^{\mathrm{b}}$ Peter G. Bourne ${ }^{\mathrm{c}}$ \\ ${ }^{a}$ Center for Genetics, Nutrition and Health, Washington, D.C., USA; ${ }^{b}$ Department of Internal Medicine \\ and Cardiology, Aarhus Sygehus University Hospital, Aarhus, Denmark; ' ${ }^{C}$ reen Templeton College, \\ University of Oxford, Oxford, UK
}

The inaugural conference on 'Healthy Agriculture, Healthy Nutrition, Healthy People' of the World Council on Genetics, Nutrition and Fitness for Health was held at Ancient Olympia, Greece, on October 5-8, 2010. The focus of the conference was on the evolutionary aspects of diet throughout the life cycle in terms of genetic predisposition, health, disease prevention and sedentary lifestyles; the dietary changes brought about by modern agriculture, agribusiness, food production systems, changes in climate and the need for urban agriculture and architecture, all of which are influenced by governments, national and international policies. Therefore, the role of governments and international organizations was extensively discussed during the last session of the conference by the participants from 20 countries representing six continents.

\section{General Conclusions and Recommendations}

- Today we live in a nutritional environment that is very different from the environments to which we are genetically adapted. Major changes in our food supply accompanied the domestication of animals and the agricultural revolution about 10,000 years ago. Later, the industrial revolution and developments in food technology brought about further major changes in the composition of foods, one of the most important of which was a change in the quantity and quality of the various fatty acids. The content of saturated fat and omega- 6 essential fatty acids increased, whereas the content of omega- 3 fatty acids decreased. A development related directly to industrialization was the intentional increase in the content of trans-fatty acids produced by the hydrogenation of polyunsatu-

The Commentary was approved by the Members of the World Council on Genetics, Nutrition and Fitness for Health who also served as the scientific committee for the Conference: M. Aksoy, E. Bababunmi, P.G. Bourne, R.C. Casper, M.J. Castillo-Garzon, G. Chrousos, R. De Caterina, M. De Lorgeril, O. Faergeman, E. Ferris, S. Gopalan, J.X. Kang, A. Leaf, F. Leighton, D. Li, H. Okuyama, L. Pérusse, A.P. Simopoulos, C. Spirou, L. Tapsell 
rated and monounsatured fatty acids. Furthermore, a 30\% decrease in the content of essential vitamins and minerals in fruits and vegetables has accompanied the use of chemical fertilizers, which are produced by processes requiring large amounts of energy and, perhaps most serious of all, resulting in dangerous disruption of the phosphorus cycle. In spite of these and many other close relationships between agriculture and food production on the one hand and nutritional and ecological problems on the other, policies for agriculture, for the environment and for human nutrition and health are largely disconnected. In our analyses, priorities and policies, we quite obviously need to take a broader view, one that at the very least recognizes the complex relationships between farming, human health, and the ecological systems on which life on earth depends.

- The presentations at the conference noted that the human genome has not changed very much during the relatively short period (10,000 years) of these major changes in diet. It is likely, therefore, that chronic diseases such as certain forms of cardiovascular disease, obesity, diabetes, cancer, arthritis, mental illness and neurodegenerative diseases are due, at least in part, to an environment of food and other elements of modern life to which we are genetically not well adapted. An analysis of epidemiological studies of dietary fatty acids and coronary heart disease (CHD) indicated that, in contrast to almost all current recommendations, high amounts of dietary linoleic acid (the major omega- 6 fatty acid) from vegetable oils actually increase the risk of CHD. The production of vegetable oils such as corn oil is a major element of modern food production systems. It may therefore be very unfortunate that foods with a high ratio of linoleic acid (omega-6) to $\alpha$-linolenic acid (omega-3) of about 15/1, common in developed countries, are now spreading to developing countries adopting the agricultural and dietary practices of the so-called developed world. Studies of transgenic animals (the FAT-1 mouse) provided further support for the concept that the effects of high linoleic acid and arachidonic acid are detrimental and that the effects of $\alpha$-linolenic acid, eicosapentaenoic acid and docosahexaenoic acid are beneficial.

- Decreasing omega- 6 fatty acids and increasing omega- 3 fatty acids in the diet, so that the ratio of omega- 6 to omega-3 is reduced from typically $15: 1$ or more to ideally $2: 1$ or less is a nutritional policy that should be considered by national and international organizations, agricultural and health associations and food industry. It is consistent with results of basic research, current epidemiology and studies of evolution. In contrast, the introduction of trans-fatty acids was not supported by any kind of research into possible health effects, and recent research indicates that trans-fatty acids confer risk of coronary heart disease at least as great as that of saturated fat. Similarly, the introduction of high amounts of omega- 6 fatty acids into the diet was supported by research that would not have met current research standards. The health effects of 'novel foods' and any dietary (quantitative and qualitative) changes contemplated by agriculturists and food technologists must therefore be carefully considered, and pilot studies should be carried out before these foods become widely available to the whole population.

- Phenylketonuria illustrates how interaction of nutrient intake with a strong but rare genetic variation determines the difference between health and disease. In contrast, much more common but weaker genetic variations, interacting with nutrients, can moderately affect health in substantial proportions of populations. As an example, metabolism of the essential nutrients, linoleic acid and $\alpha$-linolenic acid, to essential longer chain fatty acids is under the control of genes. Common variations in such genes affect the efficiency of these metabolic conversions, so that dietary requirements for linoleic and $\alpha$-linolenic acid can vary importantly between individuals. As a corollary, since the absolute and relative amounts of dietary linoleic acid and $\alpha$-linolenic acid are associated with risk of coronary heart disease and cancers such as breast cancer, risk could vary between in- 
dividuals with similar intakes of fatty acids as a function of variations in these control genes. In affluent countries, and for the rich, it is becoming possible to consider individual genetic variation in making dietary recommendations (personalized nutrition).

- Obesity, type II diabetes, and certain kinds of cardiovascular disease and cancer are commonly called diseases of 'life-style'. The term emphasizes individual rather than institutional responsibilities for avoiding disease, but in all cases the responsibility for promoting health is obviously dual. As an example, physical activity is, within wide limits, linearly related to good health, and the responsibility for adequate physical activity rests on government as well as the individual. Government must ensure the safety of physical environments so that its citizens are safe to walk and bicycle for local transportation, and government must formulate policies for education that ensure physical activity breaks for all primary and secondary education students. Urban environments must be free of pollutants, and urban architecture should encourage the involvement of citizens in activities such as local gardening of fruits and vegetables. Benefits include better citizenship, better diets, more physical activity and lower emissions of greenhouse gases.

- The enormous population of humans depends on agriculture for most of its food. Agriculture is also the most ecologically destructive of human activities, and it is an important contributor to our emissions of greenhouse gases. How destructive it is to farm depends critically on what and how we choose to farm. Producing livestock destroys ecosystems more effectively than producing fruits and vegetables, but whether we farm destructively or sustainably also depends on how intelligently we produce edible animals and plants. Allowing cows to graze rather than feeding them grains decreases the emission of methane, for example. At the same time, health depends on what and how much we eat. To continue the example, the ratio of omega- 6 to omega- 3 fatty acids is lower in milk from cows that graze than cows fed grains. Eating large amounts of red meat (from cows, hogs, sheep and goats), moreover, increases the risk of common forms of cardiovascular disease and cancer. Moderate amounts of meat and dairy, however, can be valuable sources of protein. Such complex relationships indicate that we must align our policies for producing food and for preventing disease, and future dietary recommendations must be based on what we know about ecology and climate change as well as what we know about nutrition.

- Most of the effects of global warming and climate change will make farming more difficult and food supplies more precarious. At the same time, agriculture contributes importantly to global warming and climate change, in part because contemporary agriculture, not least industrial agriculture, depends on burning fossil fuels for cheap energy. Energy in whatever form is expected to become much more expensive in the near future, however, and for this and other reasons, including environmental protection, food security, and public health, we need far more intelligent forms of agriculture. An example is the concept of agroecology as described in Olivier de Schutter's report on the right to food to the Human Rights Council of the UN in late 2010. Of equal importance, we need to integrate the results of the sciences of agriculture, nutrition and medicine. Agriculture must produce foods that we know are healthy to eat.

- Large business interests partially finance and substantially affect the relevant science. The relationship of industrial agricultural interests to agricultural universities is a case in point. Food and agriculture scientists, moreover, have traditionally driven nutrition research and the development of products that were not proven beneficial to health (i.e. high amounts of omega- 6 fatty acids in the food supply by changing animal feeds high in grains, trans-fatty acids, and high fructose syrup, etc.). Instead nutrition research and knowledge should define first what products are needed, and subsequently the food scientists and technologists grow and develop the products which should be pilot tested for 
safety and health before they are made available to the public. Nutrition departments should be leading in research and product development and Agriculture and Food Sciences and Technology departments should play a secondary role instead of leading product development before nutritional benefit to humans, animals and the environment is established. If this were to happen then the names of the various organizations should be changed from 'The Food and Nutrition Board' to 'The Nutrition, Food and Agriculture Board' indicating that nutrition research drives food and agriculture sciences research and technology. Similarly, the Food and Agriculture Organization (FAO) should change to 'Nutrition Food and Agriculture Organization'. It would serve us better if nutritional research instead were to drive food sciences.

- The complex problems of agriculture, food distribution, nutrition and human health in a setting of possibly disastrous ecosystem degradation and climate change can be addressed intelligently only by public and private policies based on science that is unbiased, critical and independent of special interests. We must therefore recognize the dangers inherent in the increasingly close ties between industry, government and university.

- To be successful the following actions are needed:

(1) Agronomic, nutritional and medical science should be independent of business interests.

(2) Need for new forms of agriculture such as agroecology.

(3) Future dietary guidelines to be based on ecological (including climatological) as well as nutritional science.

(4) Healthy oriented agriculture is needed to tailor the food chain to eradicate critical deficiencies and imbalances (e.g. change animal feeds to balance the omega-6/omega-3 fatty acid ratio).

(5) A wide range of initiatives, particularly the ones that promote active being, such as sustainable neighborhoods, smart growth, public transport, oriented developments, networks of pedestrian and bicycle friendly paths.

(6) Food sources and food pathways and how they interact or not with the built environment of cities is critical.

(7) Reduce 'food deserts' within urban environment by increasing the availability of fresh fruits and vegetables by encouraging retail outlets in such neighborhoods.

In the 5th Century B.C., Hippocrates articulated the concept of positive health as follows:

'Positive health requires a knowledge of man's primary constitution (which today we call genetics) and of the powers of various foods, both those natural to them and those resulting from human skill (today's processed food). But eating alone is not enough for health. There must also be exercise, of which the effects must likewise be known. The combination of these two things makes regimen, when proper attention is given to the season of the year, the changes of the winds, the age of the individual and the situation of his home. If there is any deficiency in food or exercise the body will fall sick.'

Today, by focusing on healthy agriculture, healthy nutrition, healthy people, we seem to develop an integrated concept contributing to a healthy world. We must now consider how the participants of the conference - concerned scientists, medical doctors, academics, health promoters, agriculturists, government policy makers and industry representatives - can actually promote the goal of healthy agriculture, healthy nutrition, Healthy people in their own countries around the world - in order that altogether could work to accelerate progress in achieving a healthy world and reflect on the role of science in the process. Ultimately, in promoting 'healthy agriculture, healthy nutrition, healthy people', we are in fact promoting a single integrated model or perception of a healthy society. Therefore, these are not three independent concepts or goals, but rather integral parts of the whole that support and sustain a healthy society and environment. 